\title{
In memoriam Marcus C. Schaub (1936-2018)
}

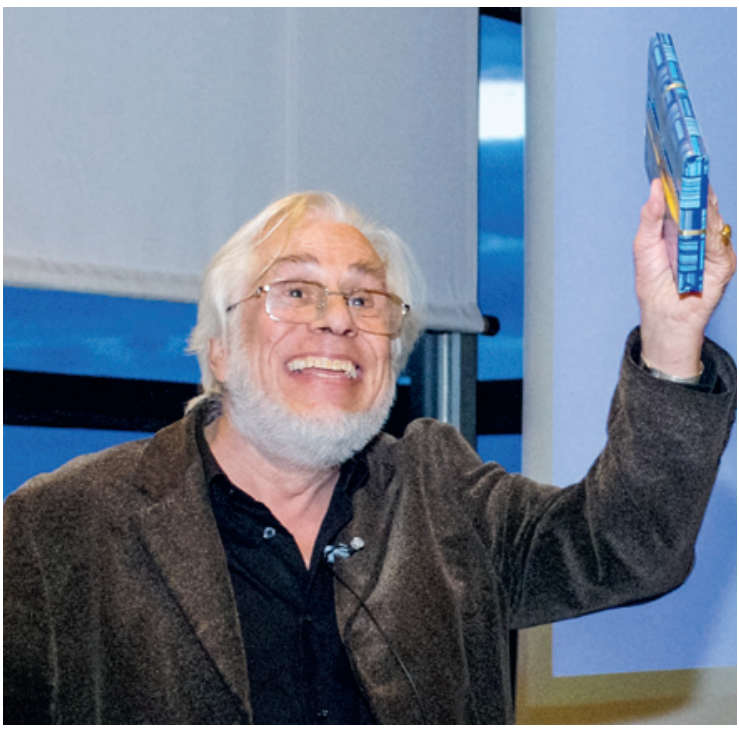

\section{Ein Leben für die Herz- und Muskel- forschung}

Am 30. Oktober 2018 ist Marcus C. Schaub im Alter von 82 Jahren verstorben. Er hinterlässt ein vielschichtiges reiches Vermächtnis auch über die Landesgrenzen hinaus, und es ist mir als sein Zögling eine Ehre, die Stationen seines Schaffens nochmals kurz in Erinnerung zu rufen. Herzlichen Dank meinen Kollegen Marijke Brink (Basel) und Claus Heizmann (Zürich), die mir dabei geholfen haben.

Marcus hatte 1961 sein Medizinstudium und medizinisches Doktorat (Dr. med.) an der Universität Basel abgeschlossen. Unbefriedigt von der - wie er selber sagte Empirie in der Medizin hat er zuerst am MaxPlanck-Institut in München in der Proteinforschung und dann von 1967-1970 bei Prof. Samuel V. Perry an der University of Birmingham in der Muskel-Biochemie geforscht und seine Dissertation (Dr. phil. nat.) verfasst. Diese Jahre im Ausland mit Ehefrau Nina und den drei kleinen Kindern, die er später als «Privileg» bezeichnete, haben den jungen wissbegierigen Arzt tief geprägt. So schreibt Marcus mit einem Augenzwinkern im Nachruf an seinen Doktorvater [1]: «He [Perry] strongly held that biochemistry is closely related to gourmet cooking» und weiter "He was mindful of my clean and punctual qualities». Eigenschaften, die von uns Schweizern im Ausland ja gerade erwartet werden und die sich beim Arbeiten mit den damals notwendigen riesigen Wassermengen zur Chromatographie mit Sephadex schliesslich auch auszahlten. Aus dieser Zeit resultierten Marcus' bahnbrechende Beiträge zur Regulation der Muskelkontraktion, die dann zusammen mit der Forschungsarbeit von Setsuro Ebashi in Tokyo und von John Gergely in Boston am Cold Spring Harbor Symposium on Muscle Contraction 1972 zum Konsensus in der Nomenklatur der Troponin-Untereinheiten geführt haben (TnI ehemals troponin-B, TnT ehemals anchoring troponin to tropomyosin, TnC ehemals troponin-A). Wie wir alle wissen, sind Troponine heute wichtige Biomarker in der Diagnose des Herzmuskelschadens und aus der Patientenbetreuung nicht mehr wegzudenken. Dies ist sicher auch den fundamentalen Arbeiten von Marcus C. Schaub zu verdanken. Trotz aller Arbeit und viel Frustration und Mühsal, die aller Forschung eigen ist, hat Marcus in seiner Zeit in Birmingham das Campus-Leben auch genossen und den halcyon British way of life kennen- und schätzen gelernt. Auch Jahrzehnte später zelebrierte Marcus die English Tea Tradition mit einer Tasse Tetley-Tee am späteren Nachmittag beim Lesen von neuen Publikationen in seinem Büro.

1970 ergab sich die Möglichkeit, in die Schweiz zurückzukehren und sich der Gruppe von Prof. Peter G. Waser am Institut für Pharmakologie und Toxikologie der Universität Zürich anzuschliessen, wo er 1980 zum Professor für Pharmakologie (mit Fakultätseinsitz) ernannt wurde. Marcus hat in dieser Funktion über 20 Jahre Generationen von Medizin- und Pharmaziestudenten mit seinen hochinteressanten und sehr didaktischen Vorlesungen geprägt. Ich weiss noch sehr genau, wie ich von seinen Pharmakologievorlesungen zur Muskelkontraktion (u.a. mit der Klärung der Frage, weshalb der Herzmuskel im Gegensatz zum Skelettmuskel keinen «Muskelkater» entwickelt) und über die Wirkungsmechanismen von Kurarepräparaten und Succhinylcholin fasziniert war, Medikamente, die ich heute als Anästhesist immer noch brauche. Nicht zu vergessen ist aber auch sein ikonisches Pharmakologie-«Repi», das er zusammen mit anderen Kollegen (Prof. Lichtensteiger/Prof. Hopf) den gestressten Studenten anbot. Marcus war ein begnadeter Redner, der sein Publikum, Studenten, Doktoranden und Kongressteilnehmer gleichermassen, in seinen Bann zie- 
hen konnte. Während nationalen Tagungen (e.g. Cardiovascular Biology und Clinical Implications Meeting in Interlaken/Murten), welche als Hauptzweck hatten, junge Wissenschaftler auszubilden und für die Forschung zu begeistern, war Marcus derjenige, der bei fast jedem Vortrag ein oder zwei schwierige Fragen gestellt hat. Für die jungen Wissenschaftler war es immer eine Herausforderung, einige haben seine Fragen gefürchtet, andere konnte er zum Denken und Forschen motivieren. Später hat Marcus erzählt, dass er sich gar nicht bewusst gewesen sei, dass seine Fragen schwierig sein könnten. Er hätte die Fragen rein aus Neugier und Interesse gestellt.

Seine Forschung am Institut für Pharmakologie konzentrierte sich zusammen mit Kollegen an der ETH (Prof. Hans M. Eppenberger) weiterhin auf kontraktile Proteine (myosin heavy and light chain isoforms), wichtige Strukturproteine (Utrophin, Dystrophin), auf Calcium in der Muskelkontraktion und auf die intrazellulären Signalwege in der Herzmuskelhypertrophie. Legendär sind seine Figuren, die das komplexe Zusammenspiel von Rezeptoren, Signalwegen und Calcium in Herzmuskelzellen darstellen [2]. Von 2000-2008 hatte ich dann das Vergnügen, mit ihm zuerst in seinem Labor am Institut für Pharmakologie und später in meinem eigenen Labor im Universitätsspital Zürich die zytoprotektiven Mechanismen volatiler Anästhetika aufzuklären [3]. Marcus hat mich immer ermuntert, die im Labor gewonnenen Resultate in der Klinik am Patienten zu testen (im Sinne der sogenannten «translational medicine»), was wir gemeinsam beim Herzschutz mittels volatiler Anästhetika bei koronarchirurgischen Patienten gemacht haben. Marcus war ein ausgezeichneter Mentor mit immensem Wissen, der sich bescheiden in den Hintergrund stellen konnte und nicht - wie andere sogenannte "Mentoren" - nur auf Publikationen stehen wollte. Er hatte auch eine Begabung, den Grundlagenforschern zu erklären, welche Forschungsthemen aus klinischer Sicht wichtig sind.

Mit zwei wichtigen Vermächtnissen für die Schweizer Herzforschung und die europäische Muskelforschung hat Marcus einen bleibenden Beitrag geleistet. Inspiriert von seinen Golden days of British muscle biochemistry, gründete Marcus 1971 die «European Society of Muscle Research (ESMR)», der er als Chairman 25 Jahre vorstand [4]. Anfänglich - so amüsierte sich Marcus zu erzählen - hiess die ESMR European Muscle Club, ein Name, der eher an einen Bodybuildingclub erinnert! Die ESMR war sich durchaus bewusst, dass Bodybuilding eine Art «angewandte Muskelforschung» ist; so wurde 1985 am Kongress in Ulm eine «atemberaubende» Bodybuilding-Showeinlage dargeboten. Aus- serdem gründete er zusammen mit drei Kollegen den «International Workshop on Cardiomyocyte Biology». Dieser Workshop wurde zum ersten Mal 1994 organisiert und findet seither alle drei Jahre im Kongresszentrum Monte Verità in Ascona statt (http://cardio ascona.ch/). Während vielen Jahren war er als einer der Hauptorganisatoren tätig, und auch in den letzten Jahren hat er sich als Scientific Board Member unermüdlich eingesetzt, damit immer höchst aktuelle Themen im Programm vertreten waren und die weltweit besten Forscher eingeladen wurden. Den Teilnehmern hat er somit einen hervorragenden Überblick über die molekulare und zellbiologische Herzforschung ermöglicht.

Marcus hatte ein extrem breit gefächertes Wissen in Wissenschaft und Medizin, hat sich aber auch für vieles andere interessiert, vor allem Theater, bildende Kunst und gesellschaftspolitische Themen. Nach jedem Kunsthaus-Besuch mit seinen guten Freunden lud Marcus alle ins Café Odeon am Bellevue ein (ein bekannter Intellektuellentreffpunkt), wo Gespräche und Diskussionen über Kunst weitergeführt wurden. Marcus und seine Gattin Nina haben sehr gerne Gäste in ihrem Zuhause bewirtet, und diese lebhaften Abende wurden von Marcus durch originelle Anektoden und Erlebnisse gewürzt. Marcus war auch ein Künstler, der selbst gemalt hat und die Bilder an gute Kollegen verschenkte!

Die von mir besonders geliebten Vorträge von Marcus waren jene, in denen er Kunst und Wissenschaft zusammenbrachte und dann die Erkenntnisse wieder zurück in gesellschaftspolitische Zusammenhänge stellte. Von diesen Vorträgen und den langen Gesprächen mit ihm über Gott und die Welt weiss ich, dass er als Vollblut-Naturwissenschaftler nichts von den «Selfish Genes» von Richard Dawkins hielt und eher ein Verfechter der «Music of Life» von Denis Noble war (siehe Bild Abschiedssymposium von Prof. C. Heizmann, 2007).

Prof. Michael Zaugg, University of Alberta, Canada

\section{Literatur}

1 Schaub MC. Golden days of British muscle biochemistry: In memoriam S. V. Perry. Journal of Muscle Research and Cell Motility. 2010;31:9-11.

2 Schaub MC, Hefti MA, Zaugg M. Integration of calcium with the signaling network in cardiac myocytes. Journal of Molecular and Cellular Cardiology. 2006;41:183-214.

3 Zaugg M, Lucchinetti E, Spahn DR, Pasch T, Schaub MC. Volatile anesthetics mimic cardiac preconditioning by priming the activation of mitochondrial K(ATP) channels via multiple signaling pathways. Anesthesiology. 2002;97:4-14

4 Schaub MC. Footsteps of the European Society for Muscle Research (1971-2008). Journal of Muscle Research and Cell Motility. 2008;29:151-4. 